\title{
Incorporating spatial dependence into a multicellular tumor spheroid growth model
}

\author{
Allen L. Garner and Y. Y. Lau ${ }^{\text {a) }}$ \\ Bioelectromagnetism Laboratory, Department of Nuclear Engineering and Radiological Sciences, University \\ of Michigan, Ann Arbor, Michigan 48109 \\ Trachette L. Jackson \\ Department of Mathematics, University of Michigan, Ann Arbor, Michigan 48109 \\ Michael D. Uhler \\ Bioelectromagnetism Laboratory, Department of Nuclear Engineering and Radiological Sciences, University \\ of Michigan, Ann Arbor, Michigan 48109 and Molecular and Behavioral Neuroscience Institute, \\ University of Michigan, Ann Arbor, Michigan 48109 \\ David W. Jordan and Ronald M. Gilgenbach \\ Bioelectromagnetism Laboratory, Department of Nuclear Engineering and Radiological Sciences, University \\ of Michigan, Ann Arbor, Michigan 48109
}

(Received 18 July 2005; accepted 8 November 2005; published online 28 December 2005)

\begin{abstract}
Recent models for organism and tumor growth yield simple scaling laws based on conservation of energy. Here, we extend such a model to include spatial dependence to model necrotic core formation. We adopt the allometric equation for tumor volume with a reaction-diffusion equation for nutrient concentration. In addition, we assume that the total metabolic energy and average cellular metabolic rate depend on nutrient concentration in a Michaelis-Menten-like manner. From experimental results, we relate the necrotic volume to nutrient consumption and estimate both the time and nutrient concentration at necrotic core formation. Based on experimental results, we demand that the necrotic core radius varies linearly with tumor radius after core formation and extend the equations for tumor volume and nutrient concentration to the postnecrotic core regime. In particular, we obtain excellent agreement with experimental data and the final steady-state viable rim thickness. () 2005 American Institute of Physics. [DOI: 10.1063/1.2146073]
\end{abstract}

\section{INTRODUCTION}

The interaction of pulsed electric fields (PEFs) with biological cells has evolved from initial applications in bacterial decontamination ${ }^{1}$ to clinical applications in electrochemotherapy and gene therapy. ${ }^{2,3}$ Most of these studies utilized PEFs below a few kilovolts per centimeter with pulse durations on the order of microseconds to milliseconds. When the combination of pulse duration and applied voltage exceeds a threshold, these pulses cause electroporation, the formation of pores in the cell membrane that permits normally impermeant molecules and ions to traverse the cell membrane. ${ }^{4}$ It is theoretically postulated that electroporation arises when the applied PEF charges the cell membrane to potentials in excess of approximately $1 \mathrm{~V} .{ }^{4}$ Recent developments in pulsed power technology have enabled the use of PEFs with much higher electric fields $(>100 \mathrm{kV} / \mathrm{cm})$ in the nanosecond pulse duration range in biological experiments. ${ }^{5,6}$ These nanosecond pulsed electric fields (nsPEFs) interact with the membranes of subcellular organelles in addition to the cell membrane, paving the way for intracellular manipulation of cells. $^{6-8}$ Researchers have observed that nsPEFs can interact with cells in a number of ways, including inducing apoptosis in cell suspensions and tumors, ${ }^{9-11}$ altering electrical

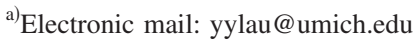

properties, ${ }^{12}$ initiating calcium release from the endoplasmic reticulum, ${ }^{11,13}$ and externalizing phosphatidylserine. ${ }^{14}$ Of particular interest is recent work involving the use of nsPEFs to treat tumors in vivo, ${ }^{15}$ which is the primary motivation for our current study into the mathematical modeling of tumor growth. One consequence of these treatments is the destruction of the vasculature supplying the tumor, which has also been observed for traditional electrochemotherapy, using microsecond to millisecond duration PEFs. ${ }^{16,17}$

While many models exist for tumor growth ${ }^{18}$ and some for angiogenesis and antiangiogenesis, ${ }^{19-29}$ a simple first question to ask is what would happen to a tumor that loses all of its vasculature due to an external stimulus (e.g., PEF exposure)? One could conjecture that such a tumor would become a large avascular tumor, whose nutrient intake by diffusion would likely be insufficient to maintain its size. Thus, conducting a mathematical analysis on the growth of an avascular tumor and its steady states becomes pertinent. Particularly interesting is recent work attempting to develop a universal law for tumor growth. ${ }^{30,31}$ This work attempts to extend traditional allometric laws for physiological processes ranging from metabolic rate to pulse rate ${ }^{32}$ to include physical and physiological bases for the parameters involved. Ultimately, coupling this type of model with other physical 
models may permit using a universal growth law to analyze cancer treatment modalities, such as recent work involving ultrashort electrical pulses to treat tumors.

Allometric laws fit the growth rate of organisms to equations of the form ${ }^{33}$

$$
\frac{d m}{d t}=a m^{x}-b m^{y},
$$

where $m$ is organism mass, $a$ and $b$ are constants, and $x$ and $y$ are generally fractional exponents. Many empirical fits using this data estimate $x$ to be $3 / 4$ and $y$ to be $1 .{ }^{32}$ Recently, West et al. have analyzed this behavior in detail, speculating that these fractional powers could arise to maximize the metabolic capacity by minimizing transport distances. ${ }^{34}$ Furthermore, they pointed out that while Eq. (1) can be used to fit many processes, it is based strictly on empirical evidence and not directly on the physical or physiological properties of the organism in question. ${ }^{35,36}$ West et al. reproduced the form of Eq. (1) from first principles. ${ }^{35,36}$ Assuming that the total-energy input to the organism must equal the sum of the energy required to maintain the organism's tissue and the energy required to create new cells allowed them to write

$$
B=\sum_{c}\left[N_{c} \beta+\epsilon \frac{d N_{c}}{d t}\right],
$$

where $B$ is the average resting metabolic rate of the whole organism, $\beta$ is the metabolic rate of a single cell, $\epsilon$ is the energy required to create a new cell, and $N_{c}$ is the total number of cells. ${ }^{35}$ Based on Ref. 34, they further assumed that $B=B_{0} m^{3 / 4}$ with $B_{0}$ a constant and $m=m_{c} N_{c}$, where $m_{c}$ is the mass of a single cell. Applying this to Eq. (2) yields

$$
\frac{d m}{d t}=a m^{3 / 4}-b m,
$$

where $a=B_{0} m_{c} / \epsilon$ and $b=\beta / \epsilon .^{35}$ While Eqs. (1) and (3) are qualitatively the same, ${ }^{36}$ their formulations differ importantly, in that the parameters $a$ and $b$ in Eq. (3) are directly related to physical parameters, while $a$ and $b$ in Eq. (1) are strictly empirically obtained parameters. This work was subsequently extended to include structures ranging in size from molecules to mitochondria, ${ }^{37}$ the effects of size and temperature on metabolic rate, ${ }^{38}$ and the effects of body size and temperature on population growth. ${ }^{39}$

Another application of this approach is to analyze the growth of avascular tumors, whose growth pattern is similar to the allometric growth observed for organisms. ${ }^{40}$ Using energy conservation as in Eq. (2) and assuming that $B=B_{0} m^{p}$ and $v=\gamma N_{c}$, where $p$ is a fractional exponent, $v$ is the volume of the tumor (the typical unit of tumor measurement), and $\gamma$ is the volume of the typical cell, ${ }^{31}$ gives

$$
\frac{d v}{d t}=a v^{p}-b v,
$$

where $a=B_{0} \gamma / \epsilon$ and $b=\beta / \epsilon$. $^{31}$

Delsanto et al. modeled the effect of nutrient depletion and mechanical stress on multicellular tumor spheroids (MTS). ${ }^{31}$ MTS are artificial tumors grown from spherical aggregations of malignant cells under strict nutritional and

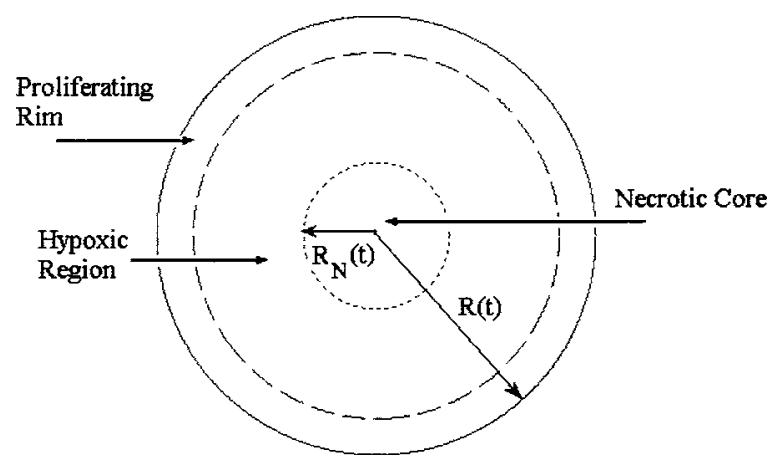

FIG. 1. Typical structure of an avascular tumor, consisting of the necrotic core $\left(r<R_{N}\right)$ and a viable region or viable rim $\left(R_{N}<r<R\right)$ containing a hypoxic region (or hypoxic rim) and a proliferating rim, where $R=R(t)$ is the overall tumor size. As a tumor grows to a critical size, $R(t)=\rho_{n}$, the cells at the center of the tumor begin to die by necrosis and the necrotic core begins to form. Not all the live cells receive sufficient nutrient to support proliferation, leading to the formation of the hypoxic region or hypoxic rim (see Refs. 40 and 57).

mechanical controls to model tumor growth. ${ }^{41-43}$ Originally grown for studies of irradiation effects on tumors, ${ }^{41-43}$ MTS have since been used for general studies of avascular tumor growth and function, including studies of nutrient supply to MTS $^{40,44}$ and mechanical stress deformation of MTS. ${ }^{45}$ Moreover, because of their relative simplicity, they have been the popular choice for mathematical modelers studying avascular tumor growth. ${ }^{31,46-49}$ MTS represent the growth dynamics of avascular tumors fairly accurately, ${ }^{40}$ including the development of a necrotic core, hypoxic region, and proliferating rim (Fig. 1), which makes them excellent model systems for this early stage of tumor growth and development that takes place until the tumors reach a few millimeters in diameter. At this point, diffusion alone is inadequate to provide sufficient nutrients to the tumor. This halts tumor growth until angiogenesis, the development of external vasculature to supply further growth. ${ }^{50}$

Because of the importance of angiogenesis and vasculature in tumor growth and metastasis, cancer researchers have focused on angiogenesis and vasculature supply to the tumor as potential targets for cancer treatment. ${ }^{16,17,50-56}$ Some of these treatments focus on specific enzymes and chemicals that inhibit angiogenesis, ${ }^{50}$ while others combine these chemicals with radiation to exploit synergistic effects. ${ }^{56}$ Particularly interesting from our perspective is the use of electrochemotherapy to destroy tumor vasculature. ${ }^{16,17}$

In Sec. II, we outline the inclusion of nutrient concentration and spatial dependence in an allometric model of MTS growth. In Sec. III, we apply this model to experimental results and consider the additional information that the model provides. We summarize the results and provide concluding remarks in Sec. IV.

\section{MODEL DEVELOPMENT}

\section{A. Reaction-diffusion models}

Traditional, first generation models for avascular tumor growth typically couple a reaction-diffusion equation for nu- 
trient concentration, $C(r, t)$, with an integrodifferential equation describing the tumor radius with respect to this nutrient to obtain ${ }^{57,58}$

$$
\begin{aligned}
& \frac{\partial C}{\partial t}=D_{C} \Delta C-\Gamma f(C), \\
& \frac{d}{d t}\left(\frac{4 \pi R^{3}}{3}\right)=4 \pi\left[\int_{R_{N}}^{R}\left[f(C)-\delta_{1}\right] r^{2} d r-\int_{0}^{R_{N}} \delta_{2} r^{2} d r\right],
\end{aligned}
$$

where $D_{C}$ is the diffusion coefficient of the nutrient, $\Delta$ is the Laplacian operator, $\Gamma f(C)$ models the rate of nutrient consumption, $R$ is the tumor radius, $R_{N}$ is the necrotic core radius with $R$ and $R_{N}$ both time-varying functions (Fig. 1), $f(C)$ is the growth rate in the viable rim due to the nutrient supply, $\delta_{1}$ is the natural death rate of the cells in the viable rim of the tumor, and $\delta_{2}$ is the decomposition rate of the cells in the necrotic core. Before the necrotic core forms, only the first integral of Eq. (6) is important and one can solve for the radius when necrotic core formation begins, $\rho_{n}$, in terms of the critical nutrient concentration $C_{n}$, and solve Eq. (6) for the corresponding time $t_{n}$. Here, and in what follows, the subscript $n$ is reserved for the specific values when the necrotic core begins to form, whereas the subscript $N$ denotes the necrotic boundary thereafter (Fig. 1). This requires enforcing the boundary conditions $C_{r}(0)=0$ and $C[R(t), t]=C_{0}$, where the subscript $r$ denotes differentiation with respect to space and $C_{0}$ is the surrounding nutrient concentration. ${ }^{57,58}$ The latter condition also applies once the necrotic core forms, i.e., the nutrient concentration on the outer boundary of the tumor equals the nutrient concentration external to the tumor. In the traditional case, this yields a third degree polynomial for $C$ in terms of $R$ and $R_{N}$ that can be combined with Eq. (6) to obtain expressions for $R$ and $R_{N}$ at steady state. ${ }^{57,58}$

\section{B. Incorporating nutrient concentration into an allometric model}

One way to incorporate spatial effects in an allometric model for MTS growth is to combine the reaction-diffusion equation for nutrient concentration with the allometric equation (4) for MTS volume rather than the integrodifferential equation (6). This additionally requires coupling (4) with (5) by including a concentration dependence in (4). The parameters $a$ and $b$ in (4) have a physical meaning with respect to $B, \beta$, and $\epsilon$, which represent cellular energies that could potentially depend on $C$ in some fashion. ${ }^{31}$ The simplest firstorder approximation is to assume that $\epsilon$, the energy required to create a new cell, is constant, while $B$ and $\beta$ depend on $C$ in a manner such that they are small when $C$ is small and plateau as $C$ becomes larger. Mathematically, expressions for $B$ and $\beta$ can be written in the same form as the MichaelisMenten equation for enzyme kinetics, ${ }^{59}$

$$
B=B_{\max } \frac{C}{F+C}=B_{0} v^{p} \frac{C}{F+C}, \quad \beta=\beta_{\max } \frac{C}{F+C},
$$

where $B_{\max }$ is the maximum average resting metabolic rate of the tumor, $\beta_{\max }$ is the maximum metabolic rate of a single cell, and $F$ is constant such that $C=F$ corresponds to $B(F)$ $=0.5 B_{\max }$ and $\beta(F)=0.5 \beta_{\max }$. Substituting Eq. (7) into Eq. (4) allows us to couple the differential equation for volume with nutrient concentration to obtain,

$$
\begin{aligned}
& \frac{\partial C}{\partial t}=D_{C} \Delta C-\kappa, \\
& \frac{d v}{d t}=\frac{\langle C\rangle}{F+\langle C\rangle}\left(a v^{p}-b v\right),
\end{aligned}
$$

where $\kappa$ represents the consumption rate of $C$ and $\langle C\rangle$ is the spatial average of nutrient concentration over the region of viable cells (Fig. 1). We define the effect of nutrient concentration in Eq. (9) by the spatial average; thus assuming that the nutrient available to all the viable cells in the tumor influences tumor growth.

To simplify the notation and reduce parameters, we shall define the following dimensionless parameters:

$$
\begin{aligned}
& \bar{R}=\frac{R}{R_{0}}, \quad \bar{v}=\frac{v}{V_{0}}=\frac{v}{(4 / 3) \pi R_{0}^{3}}, \quad \bar{C}=\frac{C}{C_{0}}, \quad \bar{t}=f_{0} t, \\
& \kappa^{*}=\frac{\kappa R_{0}^{2}}{C_{0} D_{C}}, \quad A=\frac{a V_{0}^{p-1}}{f_{0}}, \quad B=\frac{b}{f_{0}}, \quad \bar{F}=\frac{F}{C_{0}},
\end{aligned}
$$

where the bars denote the dimensionless quantities, and typical values of the scaling parameters are shown in Table I. We will omit the bars in future discussions for notational convenience. Further scaling time with respect to $b$ (i.e., $b=f_{0}$ ) or the time required for the tumor to grow to asymptotic size ${ }^{36}$ permits us to eliminate $B$ and rewrite Eqs. (8) and (9) in nondimensional form as

$$
\begin{aligned}
& \epsilon \frac{\partial C}{\partial t}=\Delta C-\kappa^{*}, \\
& \frac{d v}{d t}=\frac{\langle C\rangle}{F+\langle C\rangle}\left(A v^{p}-v\right),
\end{aligned}
$$

where $\epsilon=f_{0} R_{0}^{2} / D_{c}$. For typical parameter values (Table I), $\epsilon$ $\ll 1$ and the quasi-steady-state assumption can be used. ${ }^{57}$ For the parameters shown in Table I, $\epsilon=0.0063$ and $\kappa^{*}=27.58$.

\section{Prenecrotic core formation}

Prior to necrotic core formation (i.e., $\left.R<R_{n}\right), C_{r}(0, t)$ $=0$ and $C[R(t), t]=1$. Applying these conditions and the quasi-steady-state assumption to (11) yields

$$
C(r, t)=\frac{\kappa^{*}}{6}\left[r^{2}-R^{2}(t)\right]+1,
$$

where $r$ is nondimensional, spatial position within the tumor. To determine the spatial average of $C$ over the entire radius of the tumor, we integrate using

$$
\langle C(r, t)\rangle=\frac{1}{(4 / 3) \pi R^{3}} \int_{0}^{R} 4 \pi r^{2} C(r, t) d r
$$

to obtain 
TABLE I. Typical parameters for $0.28 \mathrm{mM}$ oxygen and $16.5 \mathrm{mM}$ glucose.

\begin{tabular}{|c|c|c|}
\hline Parameter & Value & Reference \\
\hline$A^{\prime}$ & $\begin{array}{l}6.18 \text { (prenecrotic core) } \\
1.55 \text { (postnecrotic core })\end{array}$ & $\begin{array}{l}\text { Weighted least-squares fit } \\
\text { of data from Ref. } 40 .\end{array}$ \\
\hline$b$ & $\begin{array}{c}\left.0.0016 \mathrm{~h}^{-1} \text { (prenecrotic core }\right) \\
0.0084 \mathrm{~h}^{-1}(\text { postnecrotic core })\end{array}$ & $\begin{array}{l}\text { Weighted least-squares fit } \\
\text { of data from Ref. } 40 .\end{array}$ \\
\hline$p$ & $2 / 3$ & $\begin{array}{l}\text { Diffusion limited nutrient } \\
\text { transport (Ref. 31). }\end{array}$ \\
\hline $\bar{F}$ & 0.50 & Assumption \\
\hline$R_{0}$ & $0.10 \mathrm{~cm}$ & $\begin{array}{l}\text { Typical scale of largest } \\
\text { avascular tumor (Ref. 57). }\end{array}$ \\
\hline$C_{0}$ & $16.5 \mathrm{mM}$ & $\begin{array}{l}\text { Surrounding nutrient } \\
\text { concentration (Refs. } 40 \text { and 57). }\end{array}$ \\
\hline Cell concentration & $2.01 \times 10^{8} \mathrm{cells} / \mathrm{cm}^{3}$ & Reference 61 \\
\hline$f_{0}$ & $0.0025 \mathrm{~h}^{-1}$ & $\begin{array}{l}\text { Weighted least-squares fit } \\
\text { of data from (Ref. 40). }\end{array}$ \\
\hline$D_{C}$ (glucose) & $1.1 \times 10^{-6} \mathrm{~cm}^{2} / \mathrm{s}$ & Reference 62. \\
\hline$\kappa($ glucose $)$ & $24.9 \times 10^{-17} \mathrm{~mol} /$ cell-second & Reference 44. \\
\hline
\end{tabular}

$$
\langle C(r, t)\rangle=1-\frac{\kappa^{*}}{15} v^{2 / 3}
$$

where $v=R^{3}$ is the dimensionless volume of the tumor.

Substituting Eq. (14) into Eq. (12) and defining a universal volume $u$ as $u=z v$, where $z=\left(\kappa^{*} / 15\right)^{3 / 2}$, yields

$$
\frac{d u}{d t}=\frac{1-u^{2 / 3}}{F+\left(1-u^{2 / 3}\right)} \quad\left(A^{\prime} u^{p}-u\right)
$$

where $A^{\prime}=A z^{1-p}$ and we have reduced the volumetric equation to two free parameters. In addition to steady states at $u=0$ and $u=\left(A^{\prime}\right)^{1 /(1-p)}$, which Eq. (4) shared in form, Eq. (15) now has an additional steady state at $u=1$, at which the radius is designated $R=R_{1}$. To interpret the meaning of the additional steady state, we determine the corresponding tumor size and compare it to the data from Freyer and Sutherland. ${ }^{40}$ Table I shows the typical values of the parameters required to estimate $R_{1}$ for $u=1$ with $0.28 \mathrm{mM}$ oxygen and $16.5 \mathrm{mM}$ glucose assuming that glucose is the nutrient we are considering. Using these values gives $\kappa^{*}=27.58$ and $R_{1}=0.737\left(R_{1}=737 \mu \mathrm{m}\right.$ in dimensional units $)$ while the $A^{\prime}$ dependent steady state gives $u=237 \gg 1$.

While the tumor size at the $u=1$ steady state is dramatically smaller than the final steady-state size of the tumor predicted from the prenecrotic core data, it is on the same order of magnitude as $\rho_{n}$, the tumor radius at which the necrotic core starts to form. ${ }^{40}$ For $0.28 \mathrm{mM}$ oxygen and $16.5 \mathrm{mM}$ glucose, $R_{1} \sim 2.8 \rho_{n}$. Assuming that the volume at which the necrotic core forms is given by $4 \pi \rho_{n}^{3} / 3$ in dimensional units, ${ }^{60} u_{n}=0.047$. While $R_{1} / \rho_{n}$ depends on nutrient concentration, similar relationships can be derived for the other nutrient concentrations studied by Freyer and Sutherland. This allows us to plot experimental data for $\rho_{n}$ vs $R_{1}$ to obtain the following empirical relationship for $u_{n}$ as a function of $\kappa^{*}$ :

$$
u_{n}=0.0331\left(\frac{\kappa^{*}}{15}\right)^{0.5724}
$$

Thus, Eq. (16) gives the necrotic volume as a function of normalized nutrient consumption rate and implicitly as a fraction of the steady state at $u=1$. Using this relationship, $u_{n}=0.047$ for $0.28 \mathrm{mM}$ oxygen and $16.5 \mathrm{mM}$ glucose. The numerical solution to Eq. (15) is only valid until $u=u_{n}$ or until $t=t_{n}$, beyond which a new relationship for $\langle C\rangle$ must be applied. In first generation models of avascular tumor growth, $t_{n}$ can be obtained analytically; ${ }^{57,58}$ however, introducing the more complicated expression for $\langle C\rangle$ into Eq. (12) prohibits this direct approach.

As in the case of the traditional, first generation of avascular tumor models, we can predict $C_{n}$, the nutrient concentration at which necrotic core formation begins in the tumor center $(r=0)$, from Eq. (14). Noting that $v_{n}=\left(u_{n} / z\right)^{1 / 3}$ and $v_{n}=R_{n}^{3}$ in nondimensional units, and applying the empirical relationship in Eq. (16) yields

$$
C_{n}=1-0.0917\left(\kappa^{*}\right)^{0.382} .
$$

Substituting the parameters from Table I gives $C_{n}=0.674$, the normalized critical nutrient concentration for necrotic core formation. (The external nutrient concentration is 1 in these normalized units.)

\section{Postnecrotic core formation}

As mentioned in Sec. II C, $C(r, t)$ changes mathematical form after the necrotic core forms because the boundary conditions change. Thus, we must now enforce boundary conditions inside and at the boundary of the necrotic core in addition to the boundary of the tumor. For the necrotic core $\left(0<r<R_{N}\right)$, the boundary condition (BC) is

$$
C(r, t)=C_{n}, \quad r \leqslant R_{N},
$$

which states that $C$ inside the necrotic core is at the level of the critical concentration required to initiate necrosis. In the viable rim $\left[R_{N}<r<R(t)\right]$, the $\mathrm{BCs}$ are given by 


$$
\begin{aligned}
& C[R(t), t]=1, \\
& C_{r}\left[R_{N}(t), t\right]=0 .
\end{aligned}
$$

Applying the BCs in Eqs. (18) and (19) and the quasi-steadystate assumption to Eq. (12) yields

$$
C(r, t)=\frac{\kappa^{*}}{6}\left[r^{2}-R^{2}(t)\right]+\frac{R_{N}^{3}(t) \kappa^{*}}{3}\left[\frac{1}{r}-\frac{1}{R(t)}\right]+1,
$$

where $r$ represents spatial position $\left(R_{N}<r<R\right)$ and $R_{N}(t)$ represents the growing necrotic core radius as a function of time. By demanding that Eq. (20) yields $C_{n}$ when $r=R_{N}$, we obtain a cubic polynomial relating $R$ and $R_{N}$. Instead of using this (complicated) relation, for simplicity and without sacrificing much accuracy, we use instead the empirical relation, Eq. (22) below, to evaluate the average nutrient concentration.

As in the prenecrotic core case, we average Eq. (20) over space; however, in this case, we are interested only in $C(r, t)$ in the viable rim of cells, so we use

$$
\langle C(r, t)\rangle=\frac{1}{(4 / 3) \pi\left(R^{3}-R_{N}^{3}\right)} \int_{R_{N}}^{R} 4 \pi r^{2} C(r, t) d r
$$

to obtain

$$
\begin{aligned}
\langle C(r, t)\rangle= & \frac{1}{\left(R^{3}-R_{N}^{3}\right)}\left[-\frac{\kappa^{*} R^{5}}{15}+\frac{\kappa^{*} R_{N}^{3} R^{2}}{3}-\frac{3 \kappa^{*} R_{N}^{5}}{5}\right. \\
& \left.+\frac{\kappa^{*} R_{N}^{6}}{3 R}+R^{3}-R_{N}^{3}\right],
\end{aligned}
$$

where $R$ and $R_{N}$ remain functions of time.

Equations (20) and (21) provide expressions for $C$ and $\langle C\rangle$ as functions of two unknowns: $R$ and $R_{N}$. In first generation avascular tumor models, ${ }^{57,58}$ these terms could be resolved analytically by using the integrodifferential equation for $R$ given by Eq. (6) in nondimensional units to eliminate $R_{N}$. However, as in Sec. II C, combining the reactiondiffusion relationship with an allometric equation including the dependence on $C$ prevents using an analytic approach to eliminate the additional variable. Thus, we again appeal to the experimental results of Freyer and Sutherland, where we observe that $R_{N}$ varied linearly with $R$ for all combinations of glucose and oxygen concentrations that they studied. ${ }^{40} \mathrm{By}$ drawing a best-fit line through the data for viable rim growth for $0.28 \mathrm{mM}$ oxygen and $16.5 \mathrm{mM}$ glucose, we can relate $R_{N}$ to $R$ in nondimensional units by

$$
R_{N}=1.065 R-0.275 .
$$

Substituting Eq. (22) into Eq. (21) and using the relationship $u=z R^{3}$, allow us to write $\langle C\rangle$ as a function of the universal volume $u$.

Note that the expression in Eq. (21) is much more complicated than the corresponding relation in Eq. (14) and becomes even more complicated once it is written strictly in terms of $u$. Therefore, it is not necessarily obvious from inspection whether $\langle C\rangle /(F+\langle C\rangle)$ has a zero, as in the prenecrotic core case. Thus, we plotted $H=\langle C\rangle /(F+\langle C\rangle)$ vs $R$ for $0.28 \mathrm{mM}$ oxygen and $16.5 \mathrm{mM}$ glucose from $\rho_{n}(=0.258)$ to

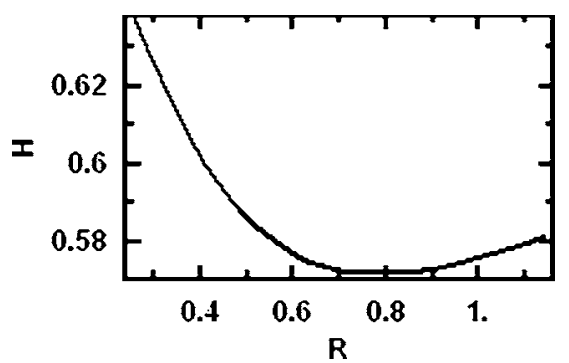

FIG. 2. $H$ vs $R$, where $H=C /(F+C)$ following necrotic core formation and $R$ is the nondimensional radius of the tumor. Note that $H$ has no zeros for $\rho_{n}<R<R_{\mathrm{SS}}$, the range of the abscissa, meaning that this term does not introduce a third steady state into Eq. (12).

$R_{\mathrm{SS}}(\sim 1.14$ based on our fit of the data from Ref. 40$)$ in Fig. 2 , where $R_{\mathrm{SS}}$ is the steady-state radius of the tumor. Figure 2 shows that this term does not have a zero in the viable rim region; therefore, it does not introduce a third steady state as in the prenecrotic core case.

The final steady-state size of the tumor is determined by the steady state at $\left(A^{\prime}\right)^{1 /(1-p)}$ while the time to reach the steady state is determined by $b$, which is physically related to the metabolic rate of the cells. The structural and physiological changes introduced by the necrotic core, as illustrated mathematically by the change in the form of $C$, will also lead to changes in $a$ and $b$. While changes in $a$ and $b$ will be absorbed directly by changes in $A^{\prime}$, changes in $b$ will also effect the time normalization, so combining the models for pre- and postnecrotic core formation requires using dimensional time.

Particularly interesting at the final steady state (SS) is the behavior of $C$. From Freyer and Sutherland, the viable rim is $\sim 0.20(200 \mu \mathrm{m})$ for this spheroid nutrient concentration and radius. Thus, if $R_{\mathrm{SS}}=1.14$, then $R_{N_{\mathrm{SS}}} \sim 0.94$ in nondimensional units. ${ }^{40}$ From Eq. (18), $C\left(R_{N}, t\right)=C_{n}$, so substituting $R(t)=R_{\mathrm{SS}}$ and $r=R_{N_{\mathrm{SS}}}$ into (20) should yield $C_{n}$ $\sim 0.674$. In fact, substituting $R_{\mathrm{SS}}$ (determined here to be $1.14)$ and $C_{n}(=0.674)$ into (20) yields $R_{N_{\mathrm{SS}}}=0.98$, which agrees well with the 0.94 estimated using Freyer and Sutherland. ${ }^{40}$

\section{MODEL APPLICATION}

Figure 3 shows the numerical solution to Eq. (15) for $u<u_{n}$ and with Eq. (21) substituted for $\langle C\rangle$ for $u>u_{n}$. Clearly, carrying out a weighted least-squares fit of the data and incorporating it into the normalized equations yield

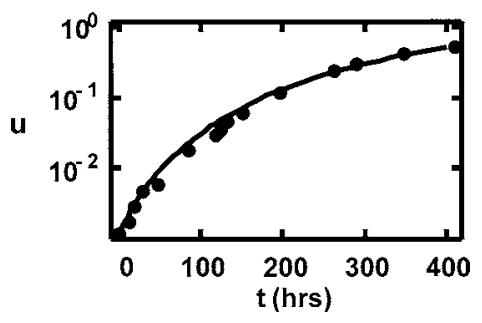

FIG. 3. Numerical solution of $u$ for $16.5 \mathrm{mM}$ glucose and $0.28 \mathrm{mM}$ oxygen. The solid circles represent experimental data (see Ref. 40) and the line represents the numerical solution of Eq. (12). 

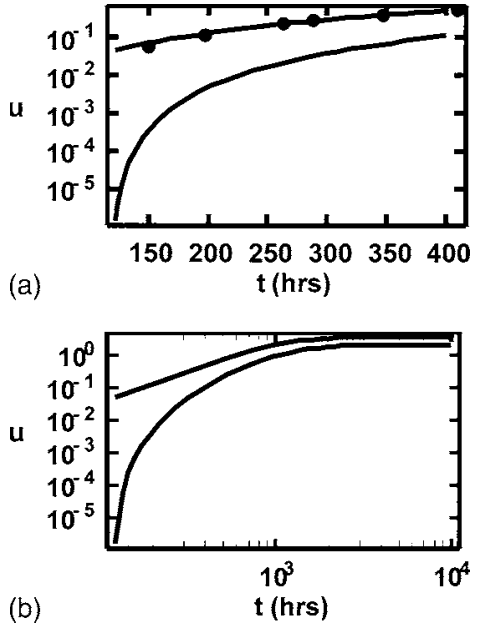

FIG. 4. Numerical solution of $u(t)$ and $u_{N}(t)$ after necrotic core formation, where the top curve represents $u(t)$ and the bottom curve $u_{N}(t)$ (a) over the range of experimental data (see Ref. 40) and (b) extrapolating to behavior at the final steady state.

physically realistic data from which physical and physiological parameters, such as $B_{0}, \beta$, and $\epsilon$, can be studied.

As mentioned earlier, $b=\beta / \epsilon$, where $\beta$ is the metabolic rate of a single cell and $\epsilon$ is the energy required to create a new cell. From the weighted least-squares fit above, $b$ $=0.0016 \mathrm{~h}^{-1}$ before necrotic core formation and $b$ $=0.0084 \mathrm{~h}^{-1}$ after necrotic core formation. Typical values for $\beta$ and $\epsilon$ are $6 \times 10^{-11} \mathrm{~W}$ (Ref. 29) and $2.0 \times 10^{-5} \mathrm{~J}^{27}$ respectively, meaning that a typical value for $b$ would be approximately $0.01 \mathrm{~h}^{-1}$, which is within an order of magnitude from the values obtained from the weighted least-squares fits. The magnitude of $b$ decreases as the tumor becomes larger (i.e., postnecrotic core) because it represents the ratio of the power required to maintain cellular function relative to the energy required to create a new cell, ${ }^{36}$ which rises as tumor size increases.

From a weighted least-squares fit of the data, $a$ $=0.0012 \mathrm{~cm} / \mathrm{h}$ before necrotic core formation and $0.00155 \mathrm{~cm} / \mathrm{h}$ after necrotic core formation. Although difficult to directly compare to the values obtained by others because of the dimensions (most growth models use $p=3 / 4$ instead of 2/3), the relative invariance of $a$ and the importance of $b$ in determining the maximum steady-state values of $u$ agree with the observations by West $e t$ al. ${ }^{36}$ These parameters allow us to calculate a maximum volume of 6.25 $\times 10^{-3} \mathrm{~cm}^{3}$, which is slightly higher, but on the same order of magnitude, as that calculated by Delsanto et al. $\left(4.4 \times 10^{-3} \mathrm{~cm}^{3}\right)$ based on the same data. Our value is lower than that estimated by Freyer and Sutherland (4.4 $\times 10^{-2} \mathrm{~cm}^{3}$ ) although it is important to note that there is a great deal of extrapolation involved in these estimations, ${ }^{40}$ which could play a role in the differences.

Figure 4(a) shows the behavior of $u$ and $u_{N}$ as a function of time after necrotic core formation compared to experimental data, ${ }^{40}$ while Fig. $4(\mathrm{~b})$ extrapolates the data to examine the steady-state behavior. As expected, we observe that the necrotic core volume initially lags the overall volume of the tumor and both reach their steady states simultaneously. These universal curves open up many options of study. For
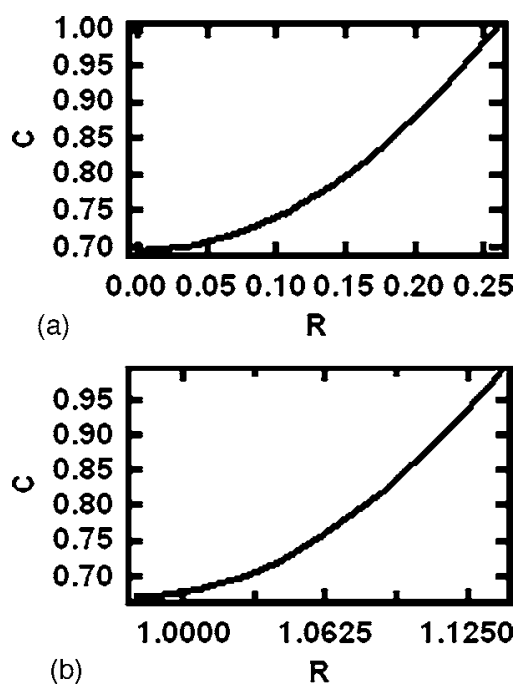

FIG. 5. (a) Nondimensional glucose concentration as a function of position at core formation (i.e., $R=\rho_{n}$ ). (b) Nondimensional glucose concentration as a function of position in the viable rim after necrotic core formation. Note that $C \approx C_{n}$ for $R \leqslant R_{N}$ and that $R_{N}=0.975$ and $R=1.127$ in this figure.

instance, although the relationship between $u$ and $u_{N}$ is based on specific concentrations of oxygen and glucose, $\kappa^{*}$ also depends on the diffusion coefficient, nutrient consumption rate, and cell concentration; therefore, one could vary $\kappa^{*}$ and observe the ensuing changes in $u$ and $u_{N}$ to obtain further insight into necrotic core formation and development.

Figure 5(a) shows the glucose concentration as a function of position within the tumor when $R=\rho_{n}$. Note that $C$ grows monotonically from $C_{n}$ at $u=0$ to 1 at $u_{n}$. Figure $5(\mathrm{~b})$ shows the glucose concentration in the viable rim once the final steady state is reached. Note that $C=C_{n}$ inside the necrotic core and at the necrotic core boundary and rises to 1 at the tumor boundary, as required by the $\mathrm{BC}$. As in the case of prenecrotic core behavior, $C$ rises monotonically. Note that the behavior of nutrient concentration in the viable rim is similar to that in the tumor once the necrotic core starts to form.

\section{CONCLUDING REMARKS}

We have extended the simple first-principles approach to modeling tumor size $\mathrm{s}^{30,31}$ to account for spatial effects, including the development of the necrotic radius, by making four primary assumptions: (1) $B$ and $\beta$ depend on nutrient concentration by a $C /(F+C)$ ratio. (2) Nutrient concentration can be modeled by a reaction-diffusion equation. (3) $R_{N}$ varies linearly with $R$ from empirical evidence. (4) $u_{N}$ varies with $C_{0}$ from empirical evidence.

Although our model is not based completely on first principles, the final results can be interpreted based on actual physiological parameters of the cell from the initial conservation of energy relationship. ${ }^{30,35}$ Unlike traditional allometric equations, the parameters that we obtain from a weighted least-squares algorithm can be related to $B, \beta$, and $\epsilon$, which have physical meanings with regard to energy input and energy usage in the cell. The increase in $b$ observed after necrotic core formation indicates a change in $\beta$ and/or $\epsilon$ that is consistent with the tumor redirecting its available energy for 
cell creation to metabolic processes. The relative invariance of $a$ is consistent with observations of West $e t a l$. and suggests that the total ratio of metabolic energy to the energy necessary to create a cell is relatively constant. Another point in this model is that it is additionally possible for changes in nutrient consumption rate, diffusion coefficient, and cell concentration to alter $A^{\prime}$, which effects the final steady-state volume of the tumor. We have shown that the values we have obtained for $a$ and $b$ for $0.28 \mathrm{mM}$ oxygen and $16.5 \mathrm{mM}$ glucose are physically realistic and lead to a final steadystate volume $\left(6.25 \times 10^{-3} \mathrm{~cm}^{3}\right)$ that is lower than that estimated by Freyer and Sutherland ${ }^{40}\left(4.4 \times 10^{-2} \mathrm{~cm}^{3}\right)$ and on the same order of magnitude as that estimated by Delsanto et al. ${ }^{31}\left(4.4 \times 10^{-3} \mathrm{~cm}^{3}\right)$.

The primary motivation for this study is the current research in using PEFs in cancer treatment, including the coupling of traditional electroporation pulses with chemotherapy drugs in electrochemotherapy ${ }^{2,16,17}$ and the application of nsPEFs directly to tumors. ${ }^{9-11,15}$ In both cases, it appears that the treatments target the tumors' vascular supply to some degree, leading to antiangiogenesis and a loss of vascular supply to the tumors. Although the mechanism is currently unknown, especially in the preliminary studies of nsPEFs, it appears that the antitumor effectiveness of electrochemotherapy arises due to a combination of increased cytotoxicity of cisplatin due to tumor cell electroporation and the antivascular effect of electrochemotherapy. ${ }^{16}$ One possible explanation of the antivascular effect is that the PEFs damage the endothelial cells, causing blood flow obstruction and ischemic death. ${ }^{16}$ In the case of nsPEFs, experimental results indicate that the capillaries supplying the tumors become immediately leaky, ${ }^{15}$ suggesting that antivascularization is an important mechanism in this treatment modality.

The physiological effects following external stimulus are quite complicated and the model in its current state cannot account for the dynamic changes in the various parameters. It is interesting to note that this simplified model predicts that the initial size of the avascular tumor could be an important variable for successful treatment, particularly near the final steady-state value, agreeing with experimental observations by Freyer and Sutherland ${ }^{40}$ and Freyer. ${ }^{63}$

This model could be extended in several ways. First, more realistic behavior with regards to nutrient effects on $\beta$ and $B$ could be introduced. Recent models developed for oxygen and glucose mass transport and metabolism ${ }^{64}$ and drug transport in MTS and monolayer cultures ${ }^{65}$ may provide some insight on extending these relationships. Moreover, dynamical changes in cell density and diffusion properties would definitely impact this simple model and would be required for modeling situations where they may rapidly change, such as during tumor treatment. Work on extending the modeling of necrotic cell death, represented by $\delta_{2}$, could provide insight into the development of the necrotic core and the progression of tumor development. Being able to account for treatment effects on $\delta_{2}$, as well as the corresponding signals and chemicals related to cell death induction, could also permit predicting the effects of different treatment modalities and possibly relate physical and physiological functions to tumor growth or shrinkage. For instance, it might be possible to directly include the effect of PEFs on the tumor within this sort of universal growth model. While current modeling in electrochemotherapy has been limited to using Gompertzian models of tumor growth, ${ }^{66}$ it is possible that the current model could be modified to study nsPEF treatment of tumor growth once data is available. ${ }^{15}$ In the long term, it is also possible that this model could be used in conjunction with models considering nsPEF-induced cellular and subcellular effects, such as those by Joshi and co-workers ${ }^{67-69}$ to elucidate the mechanisms on both the macroscopic and microscopic scales.

\section{ACKNOWLEDGMENTS}

This work was supported by the Air Force Office of Scientific Research. One of the authors (A.L.G.) was supported by a National Defense Science and Engineering Graduate Fellowship sponsored by the Department of Defense. The authors thank Richard Nuccitelli, R. James Swanson, and Richard Kowalczyk for many stimulating discussions.

${ }^{1}$ A. J. H. Sale and W. A. Hamilton, Biochim. Biophys. Acta 148, 781 (1967); A. J. H. Sale and W. A. Hamilton, Biochim. Biophys. Acta 163, 37 (1968).

${ }^{2}$ D. Rabussay, N. B. Dev, J. Fewell, L. C. Smith, G. Widera, and L. Zhang, J. Phys. D 36, 348 (2003).

${ }^{3}$ P. Lefesvre, J. Attema, and D. van Bekkum, BMC Molecular Biology 3, 12 (2002).

${ }^{4}$ J. C. Weaver and Yu. A. Chizmadzhev, Bioelectrochem. Bioenerg. 41, 135 (1996).

${ }^{5}$ M. Behrend, A. Kuthi, X. Gu, P. T. Vernier, L. Marcu, C. M. Craft, and M. A. Gundersen, IEEE Trans. Dielectr. Electr. Insul. 10, 820 (2003).

${ }^{6}$ K. H. Schoenbach, R. P. Joshi, J. F. Kolb, N. Chen, M. Stacey, P. F. Blackmore, E. S. Buescher, and S. J. Beebe, Proc. IEEE 92, 1122 (2004). ${ }^{7}$ K. H. Schoenbach, S. J. Beebe, and E. S. Buescher, Bioelectromagnetics (N.Y.) 22, 440 (2001).

${ }^{8}$ E. S. Buescher and K. H. Schoenbach, IEEE Trans. Dielectr. Electr. Insul. 10, 788 (2003).

${ }^{9}$ S. J. Beebe, P. M. Fox, L. J. Rec, K. Somers, R. H. Stark, and K. H. Schoenbach, IEEE Trans. Plasma Sci. 30, 286 (2002).

${ }^{10}$ S. J. Beebe, P. M. Fox, L. J. Rec, E. L. Willis, and K. H. Schoenbach, FASEB J. 17, 1493 (2003).

${ }^{11}$ S. J. Beebe, J. White, P. F. Blackmore, Y. Deng, K. Somers, and K. H. Schoenbach, DNA Cell Biol. 22, 785 (2003).

${ }^{12}$ A. L. Garner et al., IEEE Trans. Plasma Sci. 32, 2073 (2004).

${ }^{13}$ J. Deng, K. H. Schoenbach, E. S. Buescher, P. S. Hair, P. M. Fox, and S. J. Beebe, Biophys. J. 84, 2709 (2003).

${ }^{14}$ P. T. Vernier, Y. Sun, L. Marcu, C. M. Craft, and M. A. Gundersen, FEBS Lett. 572, 103 (2004).

${ }^{15}$ R. Nuccitelli, U. Pliquett, W. Ford, X. Chen, J. Swanson, S. J. Beebe, J. F. Kolb, and K. H. Schoenbach, Mol. Biol. Cell (accepted).

${ }^{16}$ G. Sersa et al., Br. J. Cancer 87, 1047 (2002).

${ }^{17}$ M. Cemazar, C. S. Parkins, A. L. Holder, D. J. Chaplin, G. M. Tozer, and G. Sersa, Br. J. Cancer 84, 565 (2001).

${ }^{18}$ A. Friedman, Discrete Contin. Dyn. Syst., Ser. B 4, 147 (2004); J. Moreira and A. Deutsch, Adv. Complex Syst. 5, 247 (2002).

${ }^{19}$ Y. Tao, N. Yoshida, and Q. Gao, Nonlinearity 17, 867 (2004).

${ }^{20}$ N. V. Matnzaris, S. Webb, and H. G. Othmer, J. Math. Biol. 49, 111 (2004).

${ }^{21}$ B. C. Sansone, M. Scalerandi, and C. A. Condat, Phys. Rev. Lett. 87, 128102 (2001).

${ }^{22}$ M. Scalerandi and F. Peggion, Phys. Rev. E 66, 031903 (2002).

${ }^{23}$ M. Scalerandi and B. C. Sansone, Phys. Rev. Lett. 89, 218101 (2002).

${ }^{24}$ P. Hahnfeldt, D. Panigrahy, J. Folkman, and L. Hlatky, Cancer Res. 59, 4770 (1999).

${ }^{25}$ M. E. Orme and M. A. J. Chaplain, IMA J. Math. Appl. Med. Biol. 13, 73 (1996).

${ }^{26}$ M. E. Orme and M. A. J. Chaplain, IMA J. Math. Appl. Med. Biol. 14, 
189 (1997).

${ }^{27}$ M. Scalerandi, B. C. Sansone, and C. A. Condat, Phys. Rev. E 65, 011902 (2001).

${ }^{28}$ R. K. Sachs, L. R. Hlatky, and P. Hahnfeldt, Math. Comput. Modell. 33 1297 (2001).

${ }^{29}$ S. R. McDougall, A. R. A. Anderson, and M. A. J. Chaplain, Bull. Math Biol. 64, 673 (2002)

${ }^{30}$ C. Guiot, P. G. Degiorgis, P. P. Delsanto, P. Gabriele, and T. S. Deisboeck, J. Theor. Biol. 225, 147 (2003).

${ }^{31}$ P. P. Delsanto, C. Guiot, P. G. Degiorgis, C. A. Condat, Y. Mansury, and T. S. Deisboeck, Appl. Phys. Lett. 85, 4225 (2004).

${ }^{32}$ L. von Bertalanffy, Q. Rev. Biol. 32, 217 (1957).

${ }^{33}$ R. E. Ricklefs, Funct. Ecol. 17, 384 (2003).

${ }^{34}$ G. B. West, J. H. Brown, and B. J. Enquist, Science 284, 1677 (1999).

${ }^{35}$ G. B. West, J. H. Brown, and B. J. Enquist, Nature (London) 413, 628 (2001).

${ }^{36}$ G. B. West, J. H. Brown, and B. J. Enquist, Funct. Ecol. 18, 188 (2004).

${ }^{37}$ G. B. West, W. H. Woodruff, and J. H. Brown, Proc. Natl. Acad. Sci. U.S.A. 99, 2473 (2002).

${ }^{38}$ J. F. Gillooly, J. H. Brown, G. B. West, V. M. Savage, and E. L. Charnov, Science 293, 2248 (2001).

${ }^{39}$ V. M. Savage, J. F. Gillooly, J. H. Brown, G. B. West, and E. L. Charnov, Am. Nat. 163, 429 (2004)

${ }^{40}$ J. P. Freyer and R. M. Sutherland, Cancer Res. 46, 3504 (1986).

${ }^{41}$ G. Hamilton, Cancer Lett. 131, 29 (1998)

${ }^{42}$ L. A. Kunz-Schughart, Cell Biol. Int. 23, 157 (1999).

${ }^{43}$ W. Mueller-Klieser, Am. J. Physiol. 273, C1109 (1997).

${ }^{44}$ J. J. Casciari, S. V. Sotirchos, and R. M. Sutherland, J. Cell Physiol. 151, 386 (1992).

${ }^{45}$ G. Helmlinger, P. A. Netti, H. C. Lichtenbeld, R. J. Melder, and R. K. Jain, Nat. Biotechnol. 15, 778 (1997).

${ }^{46}$ R. Chignola, A. Schenetti, G. Andrighetto, E. Chiesa, R. Foromi, S. Sartoris, G. Tridente, and D. Liberati, Cell Prolif 33, 219 (2000).

${ }^{47}$ J. A. Sherratt and M. A. J. Chaplain, J. Math. Biol. 43, 291 (2001).

${ }^{48}$ A. D. Confer and M. C. Ziskin, Cancer Res. 43, 556 (1983).

${ }^{49}$ R. Chignola et al., Cell Prolif 32, 39 (1999).
${ }^{50}$ M. M. Eatock, A. Schatzlein, and S. B. Kaye, Cancer Treat Rev. 26, 191 (2000).

${ }^{51}$ N. E. Timmins, S. Dietmair, and L. K. Nielsen, Angiogenesis 7, 97 (2004).

${ }^{52}$ N. Raghunand, R. A. Gatenby, and R. J. Gillies, Br. J. Radiol. 76, S11 (2003).

${ }^{53}$ P. Hahnfeldt, D. Panigrahy, J. Folkman, and L. Hlatky, Cancer Res. 59, 4770 (1990).

${ }^{54}$ C. S. Brock and S. M. Lee, Eur. Respir. J. 19, 557 (2002).

${ }^{55}$ K. Mross, Drug Resistance Updates 3, 223 (2000).

${ }^{56}$ P. Wachsberger, R. Burd, and A. P. Dicker, Clin. Cancer Res. 9, 1957 (2003).

${ }^{57}$ H. M. Byrne, in Cancer Modeling and Simulation, edited by L. Preziosi (Chapman and Hall, Boca Raton, 2003), p. 75.

${ }^{58}$ A. C. Burton, Growth 30, 157 (1966).

${ }^{59} \mathrm{~S}$. I. Rubinow, Introduction to Mathematical Biology (Dover, Mineola, NY, 1975), pp. 49 and 50; L. Edelstein-Keshet, Mathematical Models in Biology (McGraw-Hill, New York, 1988), pp. 271-276.

${ }^{60}$ R. Chignola, A. Schenetti, G. Andrighetto, E. Chiesa, R. Foroni, S. Sartoris, G. Tridente, and D. Liberati, Cell Prolif 33, 219 (2000).

${ }^{61}$ J. J. Casciari, S. V. Sotirchos, and R. M. Sutherland, Cell Prolif 25, 1 (1992).

${ }^{62}$ J. J. Casciari, S. V. Sotirchos, and R. M. Sutherland, Cancer Res. 48, 3905 (1988).

${ }^{63}$ J. P. Freyer, Cancer Res. 48, 2432 (1988)

${ }^{64}$ J. P. Kirkpatrick, D. M. Brizel, and M. W. Dewhirst, Radiat. Res. 159, 336 (2003).

${ }^{65}$ J. P. Ward and J. R. King, Math. Biosci. 181, 177 (2003).

${ }^{66}$ D. Miklavcic, T. Jarm, R. Karba, and G. Sersa, Math. Comput. Simul. 39, 597 (1995).

${ }^{67}$ R. P. Joshi, Q. Hu, and K. H. Schoenbach, IEEE Trans. Plasma Sci. 32, 1677 (2004).

${ }^{68}$ R. P. Joshi, Q. Hu, K. H. Schoenbach, and S. J. Beebe, Phys. Rev. E 69, 051901 (2004).

${ }^{69}$ Q. Hu, S. Viswanadham, R. P. Joshi, K. H. Schoenbach, S. J. Beebe, and P. F. Blackmore, Phys. Rev. E 71, 031914 (2005). 